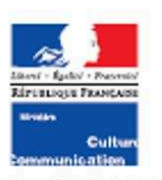

Secretariat general

Service de la

coordination des

polltiques cultureles

et de Rinnovatio

Departement

\title{
Le poids économique direct de la culture
}

Yves JAUNEAU ${ }^{*}$

\author{
The direct economic impact of culture
}

En 2011, les branches culturelles totalisent une production de 85 milliards d'euros et une valeur ajoutée de 40 milliards d'euros. La production culturelle se partage entre une partie marchande ( 69 milliards d'euros), issue de la vente des biens et services culturels des entreprises, et une partie non marchande (16 milliards d'euros), qui correspond, par convention, aux coûts de production des administrations, établissements publics et associations dans le domaine culturel.

Le poids économique direct de la culture, mesuré comme le rapport entre la valeur ajoutée des branches culturelles et celle relative à l'ensemble des branches, est de $2,2 \%$ en 2011 . Il ne prend pas en compte les retombées économiques indirectes ou induites par la culture (tourisme par exemple). Jusqu'en 2003, le poids économique direct en valeur de la culture a augmenté. Il est en recul depuis 2004, ce qui est principalement à relier au repli de l'activité dans certaines industries culturelles (édition, presse, disque).

En 2011, l'audiovisuel (radio, cinéma, télévision, vidéo, disque) concentre un quart de la valeur ajoutée culturelle. Le spectacle vivant et le patrimoine, branches majoritairement non marchandes, concentrent respectivement $18 \%$ et $11 \%$ de la valeur ajoutée. La progression au cours des quinze dernières années de la part en valeur du spectacle vivant est principalement due à l'augmentation des prix dans cette branche. À l'inverse, le livre et la presse ne concentrent plus que $15 \%$ de la valeur ajoutée culturelle en 2011 , contre $26 \%$ en 1995 . Les autres branches culturelles (agences de publicité, architecture, arts visuels, enseignement culturel) contribuent dans leur ensemble à un tiers de la valeur ajoutée culturelle.

\section{LES BRANCHES CULTURELLES : UNE PRODUCTION TOTALE DE 85 MILLIARDS D'EUROS EN 2011}

En 2011, les branches culturelles (audiovisuel, spectacle vivant, livre et presse, agences de publicité, architecture, arts visuels, patrimoine, enseignement culturel) totalisent une production de 85 milliards d'euros (tableau 1, encadré 1). Cette production se divise en trois parties (encadré 2). Tout d'abord, la production marchande culturelle (65 milliards d'euros) correspond à la production de biens et services culturels destinée à être écoulée sur le marché à un prix économiquement significatif, c'est-à-dire un prix couvrant plus de $50 \%$ des couts de production. La production pour emploi final propre (4 milliards d'euros) correspond ensuite, dans le

* Département des études, de la prospective et des statistiques. 


\section{Le poids économique direct de la culture}

The direct economic impact of culture

\section{Yves Jauneau}

Éditeur : Département des études, de la prospective et des statistiques

Lieu d'édition : Paris

Année d'édition : 2013

Date de mise en ligne : 21 septembre 2015

Collection : Culture chiffres

ISBN électronique : 9782111398641

\section{Sobooks}

http://books.openedition.org

\section{Édition imprimée}

Date de publication : 1 septembre 2013

Nombre de pages : 12

\section{Référence électronique}

JAUNEAU, Yves. Le poids économique direct de la culture. Nouvelle édition [en ligne]. Paris :

Département des études, de la prospective et des statistiques, 2013 (généré le 25 avril 2021).

Disponible sur Internet : <http://books.openedition.org/deps/496>. ISBN : 9782111398641.

(C) Département des études, de la prospective et des statistiques, 2013

Creative Commons - Attribution - Pas d'Utilisation Commerciale 3.0 non transposé - CC BY-NC 3.0 

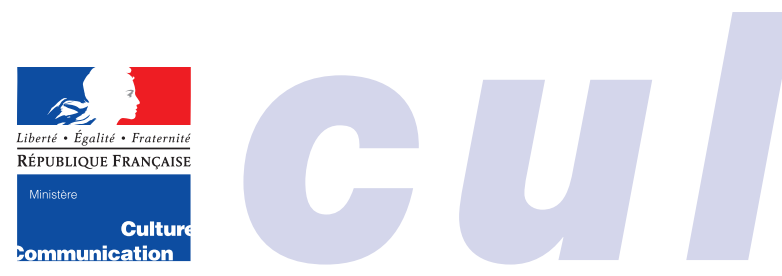

Secrétariat général

Service de la

coordination des

politiques culturelles

et de l'innovation

Département

des études,

de la prospective

et des statistiques

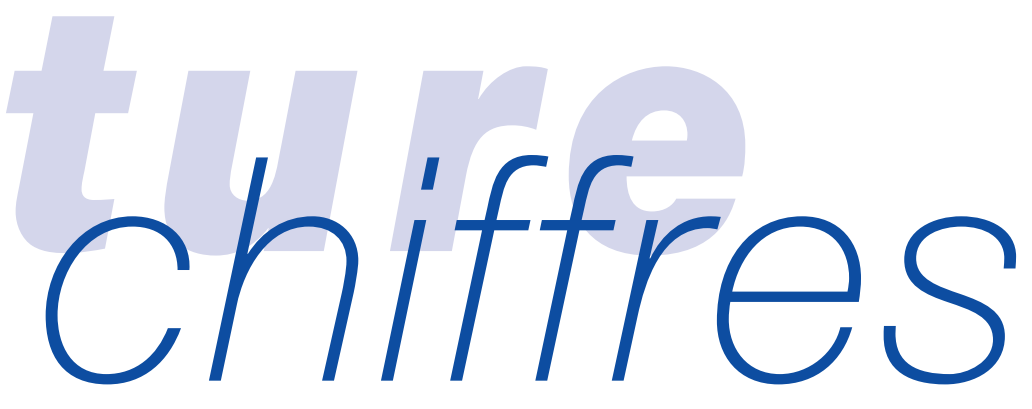

182, rue Saint-Honoré, 75033 Paris cedex 01

뚐 0140157917 - 圆 0140157999

\title{
Le poids économique direct de la culture
}

\author{
Yves JAUNEAU*
}

\section{The direct economic impact of culture}

En 2011, les branches culturelles totalisent une production de 85 milliards d'euros et une valeur ajouté de 40 milliards d'euros. La production culturelle se partage entre une partie marchande (69 milliards d'euros), issue de la vente des biens et services culturels des entreprises, et une partie non marchande (16 milliards d'euros), qui correspond, par convention, aux coûts de production des administrations, établissements publics et associations dans le domaine culturel.

Le poids économique direct de la culture, mesuré comme le rapport entre la valeur ajoutée des branches culturelles et celle relative à l'ensemble des branches, est de $2,2 \%$ en 2011 . Il ne prend pas en compte les retombées économiques indirectes ou induites par la culture (tourisme par exemple). Jusqu'en 2003, le poids économique direct en valeur de la culture a augmenté. Il est en recul depuis 2004, ce qui est principalement à relier au repli de l'activité dans certaines industries culturelles (édition, presse, disque).

En 2011, l'audiovisuel (radio, cinéma, télévision, vidéo, disque) concentre un quart de la valeur ajoutée culturelle. Le spectacle vivant et le patrimoine, branches majoritairement non marchandes, concentrent respectivement $18 \%$ et $11 \%$ de la valeur ajoutée. La progression au cours des quinze dernières années de la part en valeur du spectacle vivant est principalement due à l'augmentation des prix dans cette branche. À l'inverse, le livre et la presse ne concentrent plus que $15 \%$ de la valeur ajoutée culturelle en 2011, contre $26 \%$ en 1995. Les autres branches culturelles (agences de publicité, architecture, arts visuels, enseignement culturel) contribuent dans leur ensemble à un tiers de la valeur ajoutée culturelle.

\section{LES BRANCHES CULTURELLES : UNE PRODUCTION TOTALE DE 85 MILLIARDS D'EUROS EN 2011}

En 2011, les branches culturelles (audiovisuel, spectacle vivant, livre et presse, agences de publicité, architecture, arts visuels, patrimoine, enseignement culturel) totalisent une production de 85 milliards d'euros (tableau 1, encadré 1). Cette production se divise en trois parties (encadré 2). Tout d'abord, la production marchande culturelle (65 milliards d'euros) correspond à la production de biens et services culturels destinée à être écoulée sur le marché à un prix économiquement significatif, c'est-à-dire un prix couvrant plus de $50 \%$ des coûts de production. La production pour emploi final propre (4 milliards d'euros) correspond ensuite, dans le

* Département des études, de la prospective et des statistiques. 
cas de la culture, à une production immobilisée constituant un actif générateur de recettes ultérieures : par exemple des films ou des programmes de télévision réalisés dans l'année pour une diffusion ultérieure. Enfin, la production non marchande culturelle (16 milliards d'euros) correspond quant à elle à des biens et services culturels proposés aux ménages à un prix non économiquement significatif, car bénéficiant de l'apport de dépenses publiques (partie du budget de l'État ou des collectivités territoriales, subventions versées à des associations, aides versées par les sociétés civiles). La production non marchande est, par convention, évaluée à son coût de production (encadré 2), qui regroupe majoritairement trois types de dépenses : la rémunération des salariés, la consommation intermédiaire et les dépenses d'investissement. La production culturelle marchande est quasi exclusivement produite par les entreprises, alors que la production culturelle non marchande provient des administrations, des établissements publics ou encore des associations (encadré 2).

\section{UNE VALEUR AJOUTÉE CULTURELLE DE 40 MILLIARDS D'EUROS}

La valeur ajoutée des branches culturelles est de 40 milliards d'euros en 2011 (tableau 1). Cette valeur ajoutée correspond à la production totale culturelle diminuée des consommations intermédiaires, c'est-à-dire de l'ensemble des produits ou services transformés ou consommés au cours du processus de production (matières premières, électricité par exemple). La part de la consommation intermédiaire dans la production est, dans les branches culturelles (53\%), légèrement supérieure à la moyenne de l'économie (51\%). En effet, certaines activités culturelles, par leur activité spécifique, consomment une part importante de biens ou services de façon intermédiaire - qui ne sont donc pas comptabilisés dans la valeur ajoutée finale. C'est notamment le cas du livre et de la presse imprimés (utilisation de papier) ou de la distribution de films, de vidéogrammes ou de jeux électroniques.

\section{LE POIDS ÉCONOMIQUE DIRECT DE LA CULTURE EST DE 2,2 \% EN 2011, EN RECUL DEPUIS 2004}

Mesuré comme la part de la valeur ajoutée des branches culturelles dans l'ensemble des branches, le poids économique direct de la culture est de $2,2 \%$ en 2011 (tableau 1). Ce poids économique est relatif au périmètre harmonisé au plan européen pour délimiter les «branches culturelles » (encadré 1). Toute extension de champ par l'ajout d'activités à la frontière de l'activité culturelle (industries permettant de fabriquer des biens culturels, commerces permettant de les vendre) entraînerait une hausse du poids économique direct. En outre, ce poids économique ne prend pas ici en compte l'influence indirecte que peut avoir la culture sur d'autres activités économiques, la prise en compte de ces retombées indirectes dépendant très fortement des hypo-

\section{Graphique 1 - Poids des branches culturelles dans la valeur ajoutée de l'ensemble de l'économie, 1995-2011}

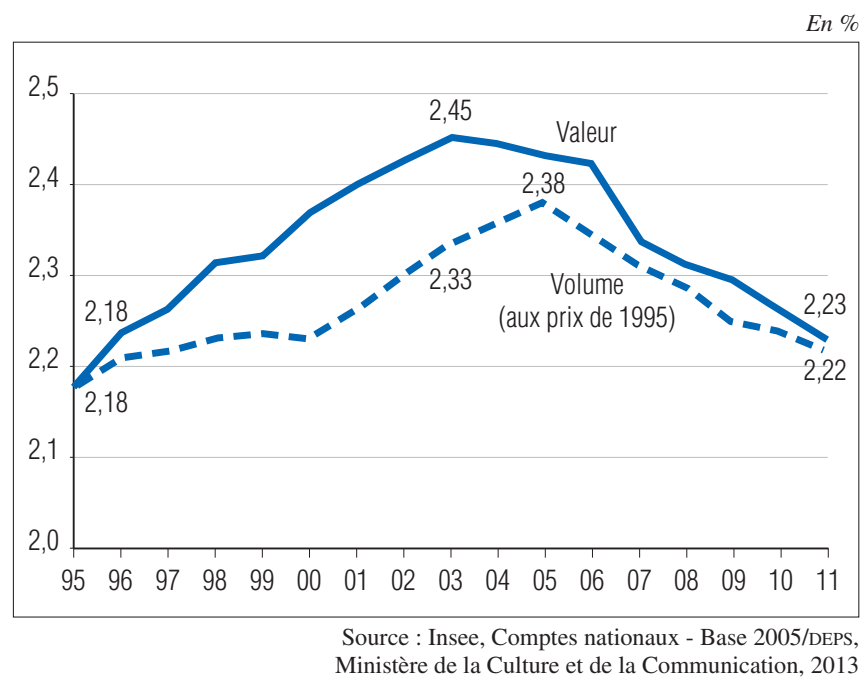

Tableau 1 - Production et valeur ajoutée dans les branches culturelles, 2011

\begin{tabular}{|c|c|c|c|}
\hline & \multicolumn{2}{|c|}{$\begin{array}{c}\text { Total par branche } \\
\text { (en milliards d'euros courants) }\end{array}$} & \multirow{2}{*}{$\begin{array}{c}\text { Poids des branches culturelles } \\
\text { dans l'ensemble des branches } \\
\text { de l'économie (en \%) }\end{array}$} \\
\hline & Ensemble de l'économie & Culture & \\
\hline Production (A) & 3655,7 & 85,0 & 2,3 \\
\hline dont : production marchande & 3011,1 & 65,2 & 2,2 \\
\hline production pour emploi final propre & 224,0 & 3,9 & 1,7 \\
\hline production non marchande & 420,6 & 15,9 & 3,8 \\
\hline Consommation intermédiaire (B) & 1861,9 & 45,0 & 2,4 \\
\hline Valeur ajoutée $(A-B)$ & 1793,8 & 40,0 & 2,2 \\
\hline
\end{tabular}


Graphique 2 - Poids des branches culturelles dans la production de l'ensemble de l'économie en valeur, 1995-2011

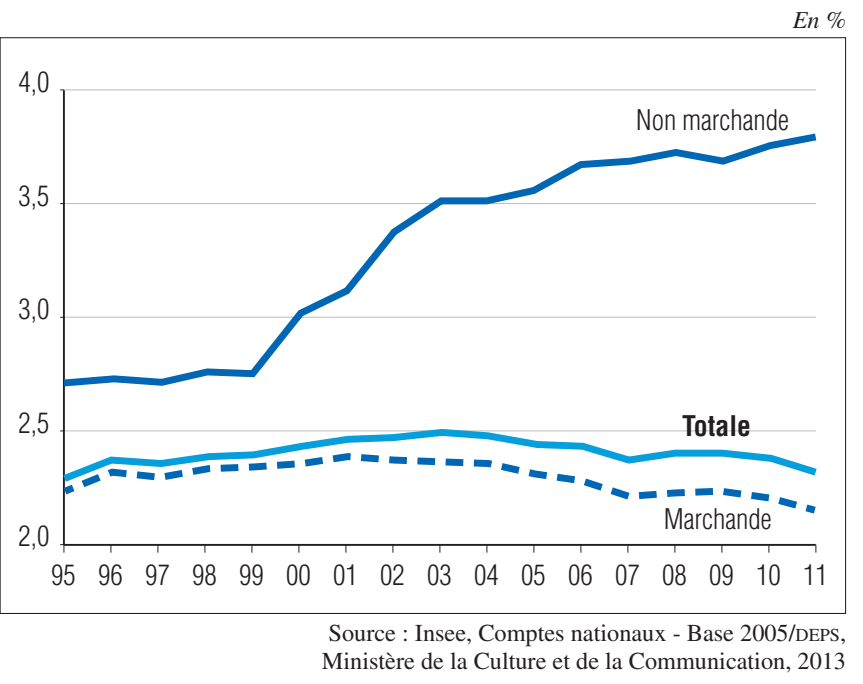

thèses faites pour les mesurer. Par exemple, le tourisme à but culturel est une partie non négligeable du tourisme. Mais prendre en compte la totalité de la valeur ajoutée de la branche des hôtels, cafés, restaurants (44 milliards d'euros) ferait varier la valeur ajoutée culturelle du simple au double. Par ailleurs, les données et les concepts manquent actuellement pour distinguer la part strictement culturelle dans le tourisme.

De 1959 à 2003, le poids économique en valeur de la culture a augmenté et culmine en 2003 à 2,4\% (graphique 1). Après cette longue période quasi ininterrompue de croissance, le poids économique de la culture diminue. C'est principalement sa composante marchande qui cause cette baisse. Dans le même temps, en effet, le poids de la culture en termes de production non marchande a constamment augmenté, passant de 2,7\% en 1995 à 3,8 \% en 2011, l'essentiel de cette croissance s'étant toutefois effectué entre 1995 et 2003 (graphique 2).

\section{BAISSE DE LA PART RELATIVE DE LA PRESSE ET DU LIVRE, HAUSSE DE L'ARCHITECTURE ET DU PATRIMOINE}

En 2011, les activités de l'audiovisuel (radio, télévision, cinéma, vidéo ou édition phonographique) constituent la principale branche d'activité culturelle en termes de poids économique, et concentrent près d'un tiers de la production totale (dont près de $40 \%$ de la production marchande) et un

\section{Graphique 3 - Répartition de la valeur ajoutée des branches culturelles en valeur, 1995-2011}

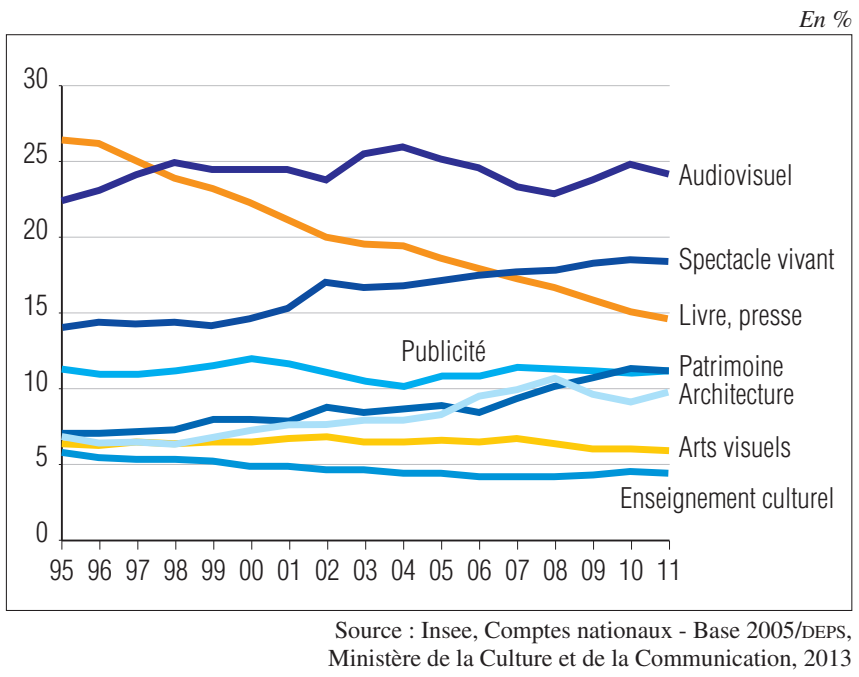

quart de la valeur ajoutée culturelle (tableau 2). Le spectacle vivant - dont $60 \%$ de la production est non marchande et valorisée à ses coûts de production ${ }^{1}$ - totalise quant à lui $18 \%$ de la valeur ajoutée culturelle, $15 \%$ de sa production et $7 \%$ de sa production marchande. Les activités du livre et de la presse regroupent $15 \%$ de la valeur ajoutée culturelle en 2011, pour $19 \%$ de sa production. Les agences de publicité, considérées ici dans leur ensemble pour l'apport créatif, même s'il s'agit d'une activité dite partiellement culturelle (encadré 1), regroupent $11 \%$ de la valeur ajoutée culturelle et les activités d'architecture $10 \%$ en 2011. Enfin, le patrimoine pèse pour $11 \%$ dans la valeur ajoutée culturelle, les arts visuels (arts plastiques, design, photographie, dont les laboratoires et les boutiques de développement photographique) $6 \%$, et l'enseignement culturel, $4 \%$.

En quinze ans, la répartition de la valeur ajoutée culturelle par branches s'est profondément modifiée. En 1995, le livre et la presse représentaient la première branche d'activité culturelle en termes de valeur ajoutée, avec $26 \%$ du total culturel (graphique 3). Ce poids a depuis régulièrement diminué, atteignant $15 \%$ en 2011. Dans le même temps, l'architecture, le patrimoine et le spectacle vivant ont vu leur poids augmenter dans l'ensemble de la valeur ajoutée. La proportion de la valeur ajoutée des autres domaines culturels (audiovisuel, publicité, enseignement culturel, arts visuels) est restée globalement stable. 
En milliards d'euros courants

\begin{tabular}{|c|c|c|c|c|c|c|c|c|}
\hline & \multicolumn{6}{|c|}{ Production } & \multicolumn{2}{|c|}{$\begin{array}{l}\text { Valeur } \\
\text { ajoutée } \\
\text { Totale }\end{array}$} \\
\hline & Valeur & $\%$ & Valeur & $\%$ & Valeur & $\%$ & Valeur & $\%$ \\
\hline \multicolumn{9}{|l|}{ Répartition par domaine culturel } \\
\hline Audiovisuel (radio, cinéma, télévision, vidéo, disque) & 27,3 & 39,4 & 0,0 & 0,0 & 27,3 & 32,1 & 9,7 & 24,2 \\
\hline Spectacle vivant & 5,2 & 7,5 & 7,3 & 45,9 & 12,5 & 14,7 & 7,4 & 18,5 \\
\hline Livre, presse & 15,9 & 23,0 & 0,0 & 0,0 & 15,9 & 18,7 & 5,8 & 14,5 \\
\hline Publicité (agences de publicité) & 8,9 & 12,8 & 0,0 & 0,0 & 8,9 & 10,4 & 4,7 & 11,8 \\
\hline Patrimoine & 0,4 & 0,5 & 6,2 & 39,0 & 6,5 & 7,7 & 4,4 & 10,9 \\
\hline Architecture & 7,3 & 10,5 & 0,0 & 0,0 & 7,3 & 8,5 & 3,9 & 9,8 \\
\hline Arts visuels (arts plastiques, design, photographie) & 3,9 & 5,7 & 0,6 & 3,9 & 4,5 & 5,3 & 2,4 & 6,0 \\
\hline Enseignement culturel & 0,3 & 0,5 & 1,8 & 11,2 & 2,1 & 2,5 & 1,7 & 4,3 \\
\hline Ensemble des branches culturelles & 69,1 & 100,0 & 15,9 & 100,0 & 85,0 & 100,0 & 40,0 & 100,0 \\
\hline
\end{tabular}

Source : Insee, Comptes nationaux - Base 2005/DEPS, Ministère de la Culture et de la Communication, 2013

\section{ArChitecture, PUblicité : LES ACTIVITÉS CULTURELLES LES PLUS TOUCHÉES PAR LA CRISE EN 2008-2009}

La branche des activités d'architecture concentre en 2011 un dixième de la valeur ajoutée culturelle, une proportion en hausse globale au cours des quinze dernières années (7\% en 1995). Le poids de l'architecture dans la valeur ajoutée culturelle a atteint $11 \%$ en 2008 , puis a reculé en 2009 et 2010, avant de légèrement remonter en 2011 (graphique 3). En effet, les activités d'architecture, plus que les autres activités culturelles, ont été largement concernées par la crise économique en 2008 et 2009. Cette branche dépend directement de l'activité du bâtiment, frappée de plein fouet par la crise. La réduction du nombre de mises en chantier de logements et de bâtiments non résidentiels a entraîné, en 2009, un recul significatif des projets architecturaux. En 2010, la branche a bénéficié de la reprise économique globale, sans néanmoins retrouver son niveau d'avant la crise.

Les activités des agences de publicité ont, quant à elles, été affectées par le recul marqué des recettes publicitaires des grands médias, passant de 9,4 milliards d'euros en 2008 à 8,1 milliards d'euros en 2009, pour légèrement remonter ensuite (8,5 milliards d'euros en 2011). De fait, le poids des agences de publicité dans la valeur ajoutée culturelle s'est stabilisé de 2008 à 2011, alors que la tendance était à l'augmentation depuis le début des années 2000.

\section{BAISSE DU POIDS DE LA CULTURE DANS L'ÉCONOMIE EN VALEUR DEPUIS 2004 ET EN VOLUME DEPUIS 2006}

Entre 1995 et 2003, les prix des branches culturelles ont augmenté de $19 \%$, contre $13 \%$ dans l'ensemble des branches de l'économie (tableau 3). Cette hausse des prix plus marquée dans les branches culturelles - particulièrement visible dans l'audiovisuel - a alors contribué à l'augmentation du poids économique de la culture en valeur.

\section{Graphique 4 - Poids de la culture dans la dépense de consommation des ménages et dans la dépense des administrations en valeur, 1995-2011}

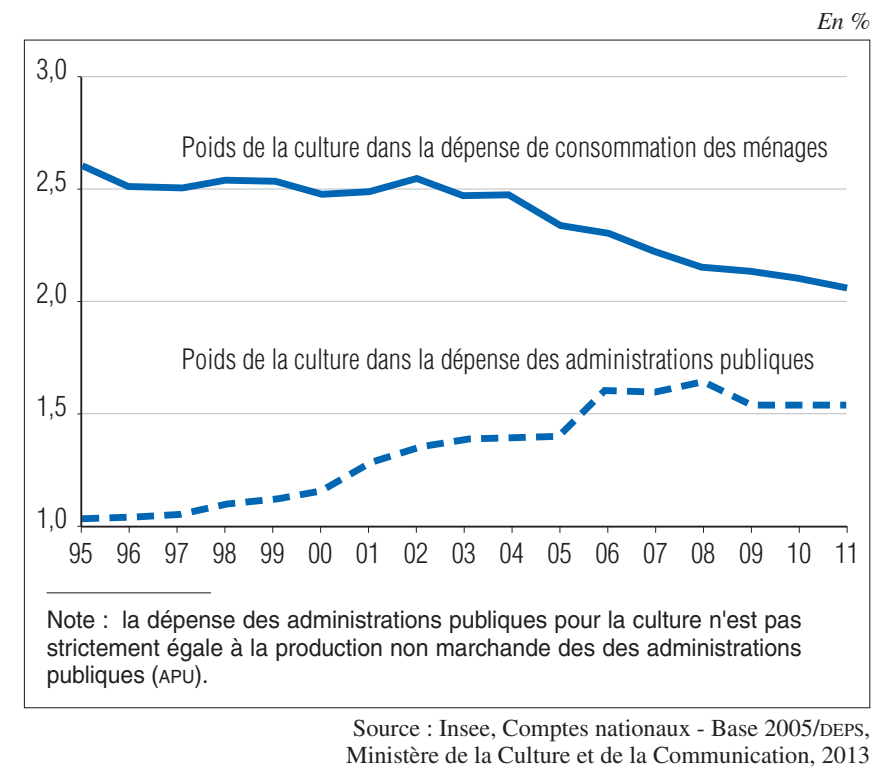


Ainsi, entre 1995 et 2003, la valeur ajoutée des branches culturelles augmente de $50 \%$ en valeur mais seulement de $26 \%$ en volume, c'est-à-dire à prix constants. Après 2003, l'effet inverse s'est produit : les prix ont en moyenne moins augmenté dans les branches culturelles (9\%) que dans le reste de l'économie (14\%). Cela est en premier lieu à relier au recul important des prix dans l'audiovisuel, et tout particulièrement dans le disque et la vidéo. La baisse, depuis 2004, du poids de la culture dans l'économie est ainsi due, dans un premier temps, à la baisse de prix de certains biens culturels. En volume, c'est-à-dire une fois neutralisé l'effet de variation des prix, le poids des branches culturelles dans l'économie baisse quand même, mais depuis 2006 seulement (graphique 1). Cette baisse en volume est particulièrement marquée, là encore, dans les branches de la presse et du livre, du spectacle vivant et des arts visuels.

\section{DEPUIS 1995, LA PART DE LA DÉPENSE DES MÉNAGES POUR LA CULTURE DIMINUE}

L'évolution du poids économique de la culture depuis 1995 - hausse puis repli - est à rapprocher de deux résultats. D'une part, les ménages ont consacré une part de plus en plus faible de leur budget à l'achat de biens culturels. Entre 1995 et 2011, les dépenses culturelles des ménages (livre, presse, audiovisuel, spectacles, etc.) n'ont augmenté en moyenne chaque année que de $1,8 \%$ en valeur, contre une progression de 3,3\% pour l'ensemble de leurs dépenses. Ainsi, le poids de la culture dans les dépenses de consommation des ménages est passé de 2,6\% à 2,1\% entre 1995 et 2011 (graphique 4). Ce recul du poids de la culture dans la dépense de consommation des ménages a pu directement impacter la production marchande des entreprises culturelles les plus fortement concernées par ce recul de la consommation (presse, livre, disque). D'autre part, la croissance des dépenses des administrations publiques pour la culture (encadré 2), qui constitue la majeure partie de la production culturelle non marchande, s'est ralentie (graphique 4). Entre 1995 et 2006, la part de la culture dans l'ensemble des dépenses des administrations publiques est passée de 1,0\% à $1,5 \%$, elle est restée stable depuis, l'effort culturel de l'État reculant légèrement (en proportion des dépenses totales), celui des collectivités territoriales se stabilisant.

\section{HAUSSE DE LA PART DES DÉPENSES DES MÉNAGES DANS LE CINÉMA ET LA TÉLÉVISION}

La part des dépenses culturelles des ménages en valeur consacrées à l'audiovisuel (hors enregistrements sonores) a régulièrement augmenté, passant de $25 \%$ en 1995 à $30 \%$ en 2011 (graphique 5). Dans les salles de cinéma, les recettes perçues au guichet ont augmenté en moyenne chaque année de $4 \%$ entre 1995 et 2011, totalisant 1,4 milliard d'euros de recettes en 2013. Les recettes publicitaires des radios et télévisions - un bon indicateur de la dynamique de l'activité dans ces secteurs - sont elles aussi restées bien orientées, augmentant de $2,3 \%$ en moyenne chaque année, malgré un léger ralentissement depuis 2003.

\section{NET RECUL DES DÉPENSES DE CONSOMMATION DE DISQUES DEPUIS 2003}

En 1995, $10 \%$ des dépenses culturelles des ménages étaient consacrés à l'achat de disques, contre $3 \%$ seulement en 2011 (graphique 5). Avec l'arrivée du téléchargement de musique sur l'internet, le marché du disque s'est effondré.

Tableau 3 - Valeur ajoutée en volume et indice des prix de la valeur ajoutée dans les branches culturelles

\begin{tabular}{|c|c|c|c|c|c|c|}
\hline & \multicolumn{3}{|c|}{$\begin{array}{c}\text { Répartition de la valeur ajoutée } \\
\text { des branches culturelles, } \\
\text { en volume (aux prix de 1995) }\end{array}$} & \multicolumn{3}{|c|}{$\begin{array}{c}\text { Évolution de l'indice } \\
\text { de prix } \\
\text { de la valeur ajoutée (\%) }\end{array}$} \\
\hline & 1995 & 2003 & 2011 & 1995-2003 & 2003-2011 & 1995-2011 \\
\hline Audiovisuel (radio, cinéma, télévision, vidéo, disque) & 22,3 & 23,4 & 27,8 & 31 & -11 & 16 \\
\hline Spectacle vivant & 14,5 & 18,9 & 17,4 & 9 & 26 & 37 \\
\hline Publicité (agences de publicité) & 11,2 & 11,1 & 14,9 & 11 & -15 & -5 \\
\hline Livre, presse & 26,1 & 20,0 & 12,8 & 14 & 29 & 46 \\
\hline Patrimoine & 7,1 & 8,3 & 10,4 & 25 & 18 & 47 \\
\hline Architecture & 6,8 & 7,8 & 9,0 & 20 & 17 & 41 \\
\hline Arts visuels (arts plastiques, design, photographie) & 6,4 & 6,9 & 5,3 & 15 & 26 & 45 \\
\hline Enseignement culturel & 5,6 & 3,9 & 3,3 & 35 & 27 & 71 \\
\hline Ensemble des branches culturelles & 100,0 & 100,0 & 100,0 & 19 & 9 & 29 \\
\hline Ensemble des branches de l'économie & & & & 13 & 14 & 29 \\
\hline
\end{tabular}


Graphique 5 - Répartition de la dépense de consommation des ménages pour la culture, 1995-2011

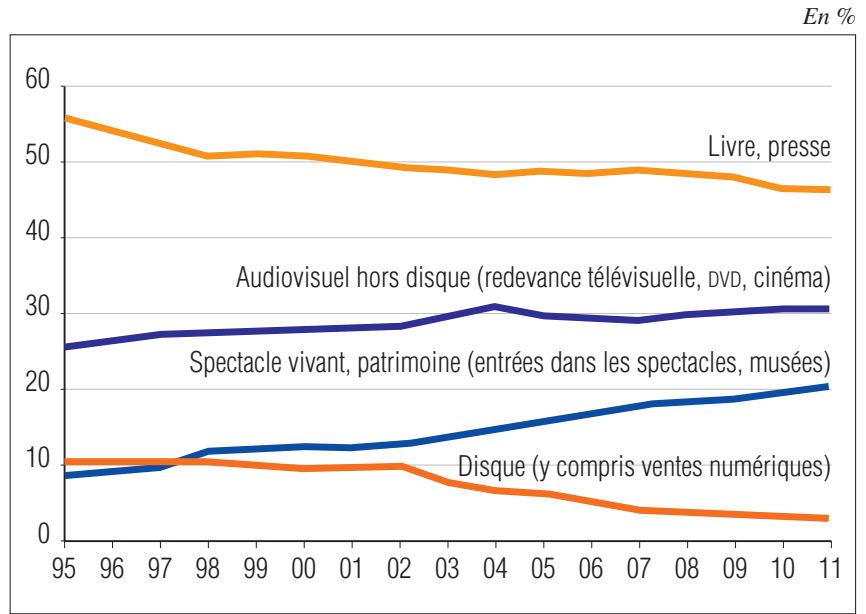

Source : Insee, Comptes nationaux - Base 2005/DEPS, Ministère de la Culture et de la Communication, 2013

Depuis 2003, le poids des activités d'enregistrement sonore et d'édition musicale dans la richesse culturelle produite a diminué de près de moitié, passant de $1,4 \%$ à $0,9 \%$ en termes de valeur ajoutée et de $2,3 \%$ à $1,5 \%$ en termes de production totale. Ainsi, en 2002, le chiffre d'affaires issu de la vente de phonogrammes par les éditeurs était de 1,3 milliard d'euros, et cette valeur a presque été divisée par quatre pour atteindre 400 millions d'euros en 2011 selon le syndicat professionnel de ce secteur (graphique 6). Le développement récent du téléchargement de musique sur des plates-formes légales semble toutefois ralentir la baisse de l'activité du secteur : en 2011, $21 \%$ du chiffre d'affaires des ventes de phonogrammes proviennent des ventes numériques, contre $13 \%$ en 2010 et seulement $3 \%$ en 2005 .

\section{BAISSE DE LA PART DES ACHATS DE JOURNAUX ET DE LIVRES DANS LES DÉPENSES CULTURELLES DES MÉNAGES}

La nette diminution de la part de la valeur ajoutée culturelle consacrée au livre et à la presse est à rapprocher du recul des pratiques au cours de cette période. Ainsi, entre 1997 et 2008, la proportion de Français âgés de 15 ans ou plus déclarant lire un quotidien au cours de l'année est passée de $73 \%$ à $69 \%$, et $36 \%$ à $29 \%$ pour ceux déclarant en lire un tous les jours. Dans la même période, la propor-

\section{Graphique 6-Chiffre d'affaires de la vente de phonogrammes par les éditeurs, 1995-2011}

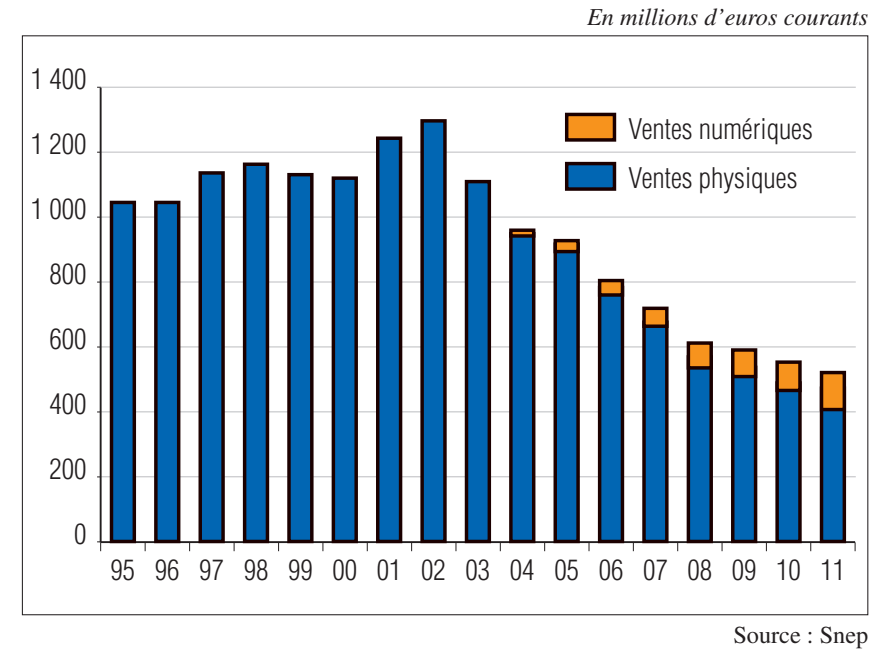

Source : Snep

tion de Français âgés de 15 ans ou plus déclarant lire un livre au cours de l'année est passée de $74 \%$ à $70 \%$, et de $37 \%$ à $31 \%$ pour ceux déclarant lire au moins 10 livres au cours de l'année. Au final, les ménages, qui consacraient en 1995 plus de la moitié de leurs dépenses culturelles à l'achat de livres ou de journaux (56\%), n'en consacrent plus que $45 \%$ en 2011 (graphique 5).

\section{LES DÉPENSES DES MÉNAGES POUR LES SPECTACLES EN HAUSSE MARQUÉE DEPUIS 1995}

Les dépenses culturelles des ménages pour les spectacles ou le patrimoine ont régulièrement augmenté au cours de la période 1995-2001, et à un rythme supérieur à celui observé pour l'ensemble des dépenses des ménages. Ainsi, en 2011, un ménage consacre $20 \%$ de ses dépenses culturelles aux activités créatives, artistiques et de spectacle ou aux bibliothèques, archives, musées, contre $8 \%$ en 1995 (graphique 5). Cette hausse soutenue, poussée par la forte hausse des prix dans le spectacle vivant, a contribué à l'augmentation de la valeur de la production marchande de ces branches. Dans le même temps, la hausse globale - malgré un ralentissement en fin de période - des dépenses culturelles des administrations publiques a contribué à augmenter le poids relatif des activités non marchandes de spectacle vivant et de patrimoine dans la valeur ajoutée culturelle. 


\section{Encadré 1 - Le champ statistique de la culture, défini de manière harmonisée au niveau européen}

Le champ de la culture est ici défini en référence à la nomenclature d'activités française (NAF), par la sélection de 29 codes parmi les 732 qui composent la nomenclature à son niveau le plus désagrégé (voir tableau ci-dessous). La définition du champ statistique de la culture a fait l'objet de travaux européens de 2009 à 2011, après que les nomenclatures nationale (nomenclature d'activités française, NAF Rév. 2) et européenne (nomenclature statistique des activités économiques dans la Communauté européenne, NACE Rév. 2) ont été rénovées en 2008. Sous l'égide d'Eurostat, l'Office statistique de l'Union européenne, un groupe de travail européen (Task Force 1) a conçu la structure du nouveau cadre statistique européen, dans un souci d'harmonisation des outils méthodologiques et pour garantir une meilleure comparabilité des résultats publiés (voir Conceptualisation statistique du champ de la culture, Paris, Ministère de la Culture et de la Communication, DEPS, coll. «Culture méthodes ", 2011-3, 2011). Ces 29 activités culturelles (au niveau 5 de la nomenclature, dit "sous-classe") sont réparties dans 8 regroupements d'activités différents, regroupements à 2 chiffres de la nomenclature appelés « divisions ». Or ces divisions sont les niveaux les plus fins disponibles pour lesquels les données comptables (valeur ajoutée, production) sont diffusées par l'Insee. II est donc nécessaire d'estimer, pour chacun des niveaux comportant au moins une activité culturelle, la part du culturel et la part du «nonculturel ».

\section{Estimation pour 2010}

Pour estimer le poids économique de la culture, on part de la production marchande donnée par la comptabilité nationale au niveau division (codes à 2 chiffres) et on la ventile au niveau de la sousclasse (codes à 5 caractères) en leur appliquant un coefficient calculé à partir des enquêtes sectorielles annuelles (ESA) de l'Insee. Ces enquêtes décomposent en effet par branche détaillée le chiffre d'affaires des entreprises de tous les secteurs d'activité. Elles permettent donc, par sommation branche par branche, d'avoir la répartition d'une branche identifiée par une division (2 caractères) en chacune des sous-classes ( 5 caractères). Cette clé de répartition est alors appliquée à la production au sens de la comptabilité nationale diffusée par division (2 chiffres). Elle diffère légèrement de la somme des productions enquêtées par l'ESA, en raison de traitements spécifiques opérés par les comptables nationaux, mais l'approximation reste raisonnable.

Pour l'enseignement culturel, on établit la production non marchande comme la somme de la dépense des centres de formation artistique (conservatoires régionaux, etc.), donnés par les Comptes de l'Éducation élaborés par la DEPP (ministère de l'Éducation nationale) et des dépenses du ministère de la Culture et de la Communication pour les établissements de l'enseignement supérieur culture (source ministérielle). Pour la production non marchande des divisions 90-91, on part de la production non marchande donnée par l'Insee (total 90-91). On répartit ce total en trois domaines (spectacle vivant, arts plastiques, patrimoine) à l'aide de plusieurs sources du ministère de la Culture (enquêtes sur les dépenses culturelles des collectivités territoriales, budgets des établissements publics culturels notamment) permettant de répartir la dépense publique culturelle (rémunérations, investissement). Cela a pour conséquence un partage de la production non marchande des divisions 90-91 légèrement différent de celui donné par l'Insee, mais a priori mieux à même d'illustrer la part proprement culturelle. Pour les ISBLSM, on répartit la production non-marchande à partir des déclarations annuelles des données sociales (DADS).

Pour calculer la valeur ajoutée, on utilise un ratio valeur ajoutée/production marchande, issu là encore des enquêtes structurelles annuelles (ESA) de l'Insee auprès des entreprises, dans les domaines totalement marchands, la valeur ajoutée ainsi estimée par sous-classe étant ensuite recalée sur la valeur ajoutée donnée par
I'Insee au niveau de chaque division. Pour les domaines partiellement non marchands (spectacle vivant, patrimoine, enseignement culturel, arts visuels), on utilise le rapport valeur ajoutée/production donné au niveau de la division par l'Insee. On estime ainsi une valeur ajoutée pour chacun des domaines culturels, dont la somme donne la valeur ajoutée des « branches culturelles » pour 2010.

Cette méthode a le triple avantage d'être relativement simple à mettre en œuvre, d'être cohérente avec les données publiées à un niveau plus agrégé par l'Insee et d'être stable dans le temps. En revanche, elle est soumise aux règles propres à la comptabilité nationale, notamment en ce qui concerne le partage marchand/nonmarchand et elle ne permet pas de prendre en compte certaines spécificités de la culture. On aurait par exemple pu lui préférer un partage entre les entreprises subventionnées et non subventionnées, ou moduler le critère des $50 \%$ des coûts de production (encadré 2).

\section{Estimation pour 1995-2009 et pour 2011}

Avec les sources et les données disponibles décrites précédemment, on a pu calculer les résultats de l'année 2010. Pour estimer le poids économique de la production marchande de 2011 et pour la période 1995-2009, on fait évoluer les coefficients culturels à partir des évolutions annuelles de chiffre d'affaires, au niveau de la sous-classe (pour chacune des sous-classes d'une division). Les données obtenues sont ensuite multipliées par un coefficient, afin d'obtenir comme total la production marchande donnée par la comptabilité nationale au niveau de chaque division. Pour l'enseignement culturel non marchand, on estime directement chacune des deux composantes (centres de formation artistique et enseignement supérieur) à partir des données annuelles des sources déjà utilisées. Pour la production non marchande des autres domaines, on actualise le partage entre spectacle vivant/arts plastiques/patrimoine en 1996 et 2002 à partir des sources précédemment évoquées, et on fait évoluer ce partage sur les autres années. Le total de production non marchande des branches 90-91 est toujours donné par les données de la comptabilité nationale. On calcule ainsi une production marchande et non marchande par domaine en 2011 et pour la période 1995-2009. Pour calculer la valeur ajoutée, on procède comme pour le calcul 2010.

\section{Estimation de la valeur ajoutée en volume}

Pour estimer la valeur ajoutée des branches culturelles en volume, on divise la valeur ajoutée en valeur estimée ci-avant au niveau le plus fin de la nomenclature d'activités par un indice de prix de valeur ajoutée. L'indice des prix utilisé provient de deux sources : indice des prix de la valeur ajoutée par branche disponible dans les comptes nationaux (au niveau division de la nomenclature), et, pour les divisions 58 et 59 , indice des prix à la consommation des ménages du produit culturel se rapprochant le plus du poste considéré (exemple: indice des prix à la consommation des journaux pour la branche $58.13 Z$ - Édition de journaux). En effet, pour ces deux divisions, il apparaît indispensable de scinder l'évolution des prix en sous-activités, certaines d'entre elles reflétant des évolutions contraires (par exemple, hausse des prix des billets de cinéma, baisse des prix des DVD ou des disques sur la période considérée).

Enfin, la valeur ajoutée par volume ainsi obtenue est multipliée par un coefficient qui permet de retrouver, par addition, le niveau exact de la valeur ajoutée en volume publiée au niveau division par l'Insee dans le cadre des comptes nationaux.

L'indice global des prix de la valeur ajoutée des branches culturelles est alors obtenu en divisant la valeur ajoutée des branches culturelles en valeur (ou à prix courants) par celle en volume (ou à prix constants). 


\begin{tabular}{|c|c|c|}
\hline \multirow[t]{2}{*}{ Niveau 2 (divisions) } & \multicolumn{2}{|l|}{ Niveau 5 (sous-classes) } \\
\hline & Activités culturelles & Activités non culturelles \\
\hline 58 - Édition & $\begin{array}{l}58.11 Z \text { - Édition de livres [LP] } \\
58.13 Z \text { - Édition de journaux [LP] } \\
58.14 Z \text { - Édition de revues et périodiques [LP] } \\
58.21 Z \text { - Édition de jeux électroniques [AV] }\end{array}$ & $\begin{array}{l}\text { 58.12Z - Édition de répertoires et de fichiers d'adresses } \\
\text { 58.19Z - Autres activités d'édition } \\
\text { 58.29A et 58.29B - Édition de logiciels } \\
58.29 \text { - Édition de logiciels applicatifs }\end{array}$ \\
\hline $\begin{array}{l}59 \text { - Production de films } \\
\text { cinématographiques, } \\
\text { de vidéo et de programmes } \\
\text { de télévision; } \\
\text { enregistrement sonore } \\
\text { et édition musicale }\end{array}$ & $\begin{array}{l}\text { 59.11A - Production de films et de programmes } \\
\quad \text { pour la télévision [AV] } \\
\text { 59.11B - Production de films institutionnels et publicitaires [AV] } \\
59.11 \text { - Production de films pour le cinéma [AV] } \\
\text { 59.12Z - Postproduction de films cinématographiques, } \\
\quad \text { de vidéo et de programmes de télévision [AV] } \\
\text { 59.13A - Distribution de films cinématographiques [AV] } \\
\text { 59.13B - Édition et distribution vidéo [AV] } \\
\text { 59.14Z - Projection de films cinématographiques [AV] } \\
\text { 59.20Z - Enregistrement sonore et édition musicale [AV] }\end{array}$ & \\
\hline 60 - Programmation et diffusion & $\begin{array}{l}\text { 60.10Z - Édition et diffusion de programmes radio [AV] } \\
60.20 \mathrm{~A} \text { - Édition de chaînes généralistes [AV] } \\
60.20 \mathrm{~B} \text { - Édition de chaînes thématiques [AV] }\end{array}$ & \\
\hline 63 - Services d'information & $63.91 Z$ - Activités des agences de presse [LP] & $\begin{array}{l}\text { 63.11Z - Traitement de données et activités connexes } \\
63.12 Z \text { - Portails Internet } \\
\text { 63.99Z - Autres services d'information }\end{array}$ \\
\hline $\begin{array}{l}71 \text { - Activités d'architecture } \\
\text { et d'ingénierie; } \\
\text { activités de contrôle } \\
\text { et analyses techniques }\end{array}$ & $71.11 \mathrm{Z}$ - Activités d'architecture [ARCHI] & $\begin{array}{l}\text { 71.12A - Activité des géomètres } \\
71.12 \mathrm{~B} \text { - Ingénierie, études techniques } \\
71.20 \mathrm{~A} \text { - Contrôle technique automobile } \\
71.20 \mathrm{~B} \text { - Analyses, essais et inspections techniques }\end{array}$ \\
\hline 73 - Publicité et études de marché & 73.11Z - Activités des agences de publicité (p) [PUB] & $\begin{array}{l}73.12 Z \text { - Régie publicitaire de médias } \\
73.20 Z \text { - Études de marché et sondages }\end{array}$ \\
\hline $\begin{array}{l}74 \text { - Autres activités spécialisées, } \\
\text { scientifiques et techniques }\end{array}$ & $\begin{array}{l}\text { 74.10Z - Activités spécialisées de design [AR] } \\
74.20 Z \text { - Activités photographiques (p) [AR] }\end{array}$ & $\begin{array}{l}\text { 74.30Z - Traduction et interprétation } \\
\text { 74.90A - Activité des économistes de la construction } \\
\text { 74.90B - Activités spécialisées, scientifiques et } \\
\text { techniques diverses }\end{array}$ \\
\hline 85 - Enseignement & $85.52 Z$ - Enseignement culturel [*] [EC] & $\begin{array}{l}\text { 85.10Z - Enseignement pré-primaire } \\
\text { 85.20Z - Enseignement primaire } \\
\text { 85.31Z - Enseignement secondaire général } \\
\text { 85.32Z - Enseignement secondaire technique ou prof. } \\
85.41 Z \text { - Enseignement post-secondaire non supérieur } \\
\text { 85.42Z - Enseignement supérieur ( }{ }^{*} \text { ) } \\
85.51 Z \text { - Enseignement du sport et des loisirs } \\
\text { 85.53Z - Enseignement de la conduite } \\
\text { 85.59A - Formation continue d'adultes } \\
\text { 85.59B - Autres enseignements } \\
\text { 85.60Z - Activités de soutien à l'enseignement }\end{array}$ \\
\hline $\begin{array}{l}90 \text { - Activités créatives, } \\
\text { artistiques et de spectacle }\end{array}$ & $\begin{array}{l}\text { 90.01Z - Arts du spectacle vivant [SV] } \\
\text { 90.02Z - Activités de soutien au spectacle vivant [SV] } \\
\text { 90.03A - Création artistique relevant des arts plastiques [AR] } \\
\text { 90.03B - Autre création artistique [AR] } \\
\text { 90.04Z - Gestion de salles de spectacles [SV] }\end{array}$ & \\
\hline $\begin{array}{l}91 \text { - Bibliothèques, archives, } \\
\text { musées et autres } \\
\text { activités culturelles }\end{array}$ & $\begin{array}{l}\text { 91.01Z - Gestion des bibliothèques et des archives [PAT] } \\
\text { 91.02Z - Gestion des musées [PAT] } \\
\text { 91.03Z - Gestion des sites et monuments historiques et } \\
\text { des attractions touristiques similaires [PAT] }\end{array}$ & $\begin{array}{l}\text { 91.04Z - Gestion des jardins botaniques et zoologiques } \\
\text { et des réserves naturelles }\end{array}$ \\
\hline \multicolumn{3}{|l|}{ (p) Code partiellement culturel. } \\
\hline \multicolumn{3}{|c|}{$\begin{array}{l}\text { (*) Le code } 85.52 Z \text { exclut les établissements d'enseignement supérieur (dont ceux relevant de la Culture), qui sont classés en code } 85.42 Z \text { (enseignement supérieur). Ces établissements (une centaine environ) son } \\
\text { ici pris en compte dans l'estimation de la valeur ajoutée. }\end{array}$} \\
\hline $\begin{array}{l}\text { Note : les activités culturelles sont regroupées } \\
\text { PAT : patrimoine; } E C \text { : enseignement culturel }\end{array}$ & $\begin{array}{l}\text { uit domaines (voir tableau } 2 \text { de cette publication), comme indiqué entre parenthèses } \\
\text { livre et presse ; AR : arts visuels ; ARCHI : architecture ; PUB : publicité). }\end{array}$ & Ins le tableau ci-avant : audiovisuel (AV : audiovisuel ; SV : spectacle vivant; \\
\hline
\end{tabular}


Une rétropolation du poids économique de la culture par «minimum/maximum » pour la période 1959-2011

Pour estimer les données avant 1995, on calcule ici non plus des taux d'inclusion mais on émet des hypothèses sur l'estimation minimale ou maximale d'inclusion de la culture. Les nomenclatures et leur niveau de détail se sont en effet modifiés en 1995 et n'existent qu'à un niveau agrégé avant 1995. Les résultats présentés ci-après montrent que le poids économique direct de la culture semble presque continuellement augmenter de 1959 à 2003, à l'exception d'un temps d'arrêt à la fin des années 1970 et au début des années 1980.

\section{Graphique 7 - Poids des branches culturelles dans la valeur ajoutée de l'ensemble de l'économie, estimation par minimum/maximum, 1959-2011}

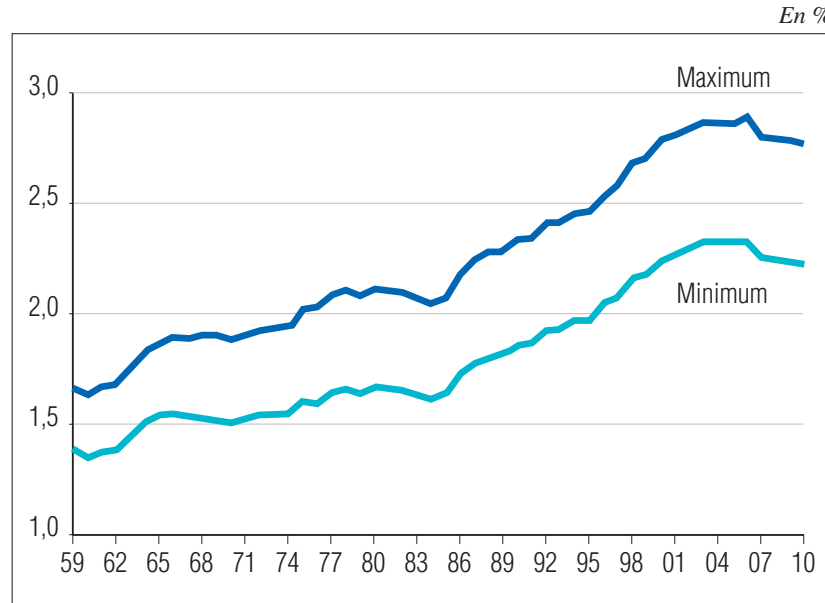

Source : Insee, Comptes nationaux - Base 2005/DEPS, Ministère de la Culture et de la Communication, 2013

\section{La mesure du poids économique de la culture est sensible à la définition de son champ}

Le champ statistique ici utilisé pour mesurer le poids économique de la culture n'intègre pas certains domaines très difficiles à cerner d'un point de vue statistique, soit parce qu'ils ne correspondent pas à une branche au niveau le plus fin de la nomenclature (galeries d'art par exemple, intégrées à la branche " commerce de détail »), soit parce qu'ils sont relatifs à des activités dites créatives mais dont la majeure partie est relative à l'industrie ou au commerce (l'industrie de l'habillement pour la mode, commerce de détail du livre). À l'inverse, le champ choisi ici intègre des champs partiellement culturels, notamment les activités des laboratoires de développements photographiques ou encore les agences de publicité, secteurs à forte valeur ajoutée, dont la suppression du champ culturel ferait diminuer sa valeur ajoutée de $10 \%$.

Ainsi, ne sont pas retenues ici des branches des activités industrielles qu'on aurait pu considérer comme culturelles comme les activités d'imprimerie (18.11 et 18.12, ce dernier code - imprimerie de labeur - ayant un contenu culturel encore plus limité), ou la reproduction (18.20) et la fabrication d'instruments de musique (32.20) car ce sont des activités qui ne permettent que la reproduction de produits culturels mais n'y apportent pas de valeur culturelle ${ }^{1}$. De même en est-il des équipements et matériaux (depuis la fabrication des optiques jusqu'à celle des peintures ou autres encres ou vernis d'imprimerie). La valeur ajoutée de la fabrication d'instruments de musique est égale, en 2010, à 96 millions d'euros et son apport au calcul du poids économique de la culture reste marginal. Inclure cette activité conduirait au risque de faire perdre sa visibilité à la culture, de noyer ses représentations dans des secteurs divers. Enfin, la production des outils de diffusion (les « contenants ", c'està-dire toutes les activités connexes de transmission et de mise à disposition de services de télécommunications ; coût d'un abonnement ADSL par exemple) n'est pas incluse dans le champ, même si ces outils permettent la diffusion d'activités et de services culturels (une connexion internet permet d'écouter de la musique en ligne par exemple ${ }^{2}$ ).

Le travail au sein du groupe de travail statistique européen pour une définition du champ culturel a résulté d'un choix consensuel de pays aux histoires et administrations pourtant différentes.

\section{Le champ culturel \\ dans la dépense de consommation des ménages}

Sont comptés comme dépenses culturelles certains postes ou regroupements de postes de la nomenclature de fonctions (col$\left.C O P^{3}\right)$, qui répartit la consommation des ménages en produits: HJ58Z1A (livres), HJ58Z1 (journal), HJ58Z1D (revues et périodiques), HJ59Z2 (films cinématographiques, vidéos et programmes de télévision), HJ59Z3 (enregistrement sonore et édition musicale), A88.60 (programmation et diffusion), A88.90 (activités créatives, artistiques et de spectacle) et A88.91 (bibliothèques, archives, musées et autres activités culturelles). En revanche, par cohérence avec le poids économique des branches culturelles, ne sont pas comptées comme dépense culturelle les dépenses de "distribution de bouquets de programmes de radio et de télévision » (classées en division 61 de la coıcoP). Ces nomenclatures sont très légèrement différentes de celles utilisées pour élaborer la production et la valeur ajoutée. II n'est donc ici pas possible d'établir une décomposition complète de la valeur ajoutée des branches culturelles en différents agrégats (dont la dépense de consommation des ménages), comme cela est fait par exemple dans l'ensemble de l'économie par la comptabilité nationale ${ }^{4}$, et d'établir ainsi un compte annexe ou « satellite " propre à la culture. Les sources ne seraient par ailleurs pas suffisantes pour estimer chacune des contreparties de la valeur ajoutée.

\section{Le champ culturel \\ dans la dépense des administrations publiques}

La nomenclature de dépenses de consommation est différente de celle de la production mais la plupart des rapprochements sont possibles. Sont comptés comme dépenses culturelles certains postes de la nomenclature internationale définie dans le système des Comptes nationaux de 1993 et révisée en 1999: la COFOG (Classification of the Functions of Government ${ }^{5}$ ): 08.2 - Services culturels, 08.3 - Services de radiodiffusion, de télévision et d'édition et 08.5 - R-D dans le domaine des loisirs, de la culture et du culte.

1. On retrouve aussi ces choix dans le cadre pour les statistiques culturelles établi par l'Unesco en 2009.

2. L'achat de biens culturels en ligne est bien en revanche dans le champ : par exemple, le téléchargement légal de musique est compris dans le code NAF $5920 Z$

- Enregistrement sonore et édition musicale).

3. Voir http://unstats.un.org/unsd/cr/registry/regcst.asp?Cl=5\&Top=2\&Lg=2

4. Voir par exemple: http://www.insee.fr/fr/themes/comptes-nationaux/tableau.asp?sous_theme=1\&xml=t 1101

5. Voir http://www.insee.fr/fr/themes/theme.asp?theme=16\&sous_theme=3.3 


\section{Encadré 2- Définitions et concepts}

\section{Les acteurs ou secteurs institutionnels}

Cinq grands secteurs institutionnels composent l'Économie nationale : les sociétés non financières (SNF - S.11), les sociétés financières (SF - S.12), les administrations publiques (APU - S.13), les ménages (S.14), les institutions sans but lucratif au service des ménages (ISBLSM - S.15). L'ensemble des unités non résidentes, dans la mesure où elles entretiennent des relations économiques avec des unités constituant l'Économie nationale, sont regroupées dans le Reste du monde (S.2). Dans la culture, trois secteurs institutionnels sont concernés (S.11, S.13, S.15).

Les sociétés non financières produisent des biens et services marchands (c'est-à-dire à un prix couvrant plus de $50 \%$ des coûts de production) et n'ont pas de production non marchande (voir plus loin). En revanche, les deux autres acteurs (APU et ISBLSM) produisent très majoritairement des biens et services non marchands, c'est-à-dire à un prix couvrant moins de $50 \%$ des coûts de production. Le critère de $50 \%$ est une convention de la comptabilité nationale, tout comme le partage entre APU et ISBLSM. II permet notamment de disposer d'une comptabilité publique comparable entre pays européens. Si le seuil de $50 \%$ était abaissé, il y aurait moins d'établissements classés comme non marchands, ce qui ferait diminuer le total de la production non marchande puisqu'on ne tiendrait compte, pour ces établissements-là, que de leur production marchande.

\section{Sociétés non financières, appelées ici entreprises}

Les entreprises regroupent les agents économiques dont la fonction principale est de produire des biens et des services non financiers marchands. Elles composent la plus grande partie du système productif. Elles se répartissent en deux catégories principales:

- les sociétés non financières, qui ont le plus souvent une personnalité juridique propre (sociétés anonymes (SA, SARL, SAS), entreprise unipersonnelle à responsabilité limitée (EURL), coopératives, sociétés de personnes, établissements publics à caractère industriel et commercial (EPIC), associations sans but lucratif, holdings, etc.), et une comptabilité distincte de celle de leur propriétaire ;

- les entreprises individuelles dont la personnalité juridique n'est pas différente de celle de leur propriétaire (artisan, commerçant, profession libérale, etc.). Le statut juridique d'une entreprise ne doit pas être confondu avec son régime qui concerne uniquement le type d'imposition fiscale : ainsi on parle du régime de l'auto-entrepreneur, du régime de la micro-entreprise.

\section{Administrations publiques (APU)}

Ensemble des unités institutionnelles dont la fonction principale est de produire des services non marchands ou d'effectuer des opérations de redistribution du revenu et des richesses nationales. Elles tirent la majeure partie de leurs ressources de contributions obligatoires. Le secteur des administrations publiques comprend les administrations publiques centrales (y compris les organismes divers d'administration centrale - ODAC - qui regroupent des organismes auxquels l'État a donné une compétence fonctionnelle spécialisée au niveau national), les administrations publiques locales et les administrations de sécurité sociale.

Dans la culture, les APU sont: l'État (qui consacre une partie de son budget à la culture), les ODAC (certains établissements publics ou établissements subventionnés) et les collectivités territoriales (qui consacrent une partie de leurs dépenses à la culture). Les ODAC culturels sont les établissements publics sous tutelle du ministère (Opéra national de Paris, musée du Louvre, écoles d'enseignement supérieur Culture, etc.) ainsi que certains grands établissements subventionnés (théâtres nationaux, orchestres). La liste des ODAC est disponible ici : http://www.insee.fr/fr/indicateurs/cnat_annu/ base_2005/methodologie/odac-simple.pdf. La plupart des établissements culturels subventionnés sont toutefois classés par la comptabilité nationale en ISBLSM (voir ci-après).
Institutions sans but lucratif au service des ménages (ISBLSM)

Elles regroupent l'ensemble des unités privées dotées de la personnalité juridique qui produisent des biens et services non marchands au profit des ménages. Leurs ressources principales proviennent de contributions volontaires en espèces ou en nature effectuées par les ménages en leur qualité de consommateurs, de versements provenant des administrations publiques, ainsi que de revenus de la propriété.

Dans la culture, outre les ODAC précédemment mentionnés, les ISBLSM sont quasi exclusivement des associations, et sont présentes dans quatre domaines: l'enseignement culturel (école de musique territoriale par exemple), le spectacle vivant (compagnie théâtrale par exemple), le patrimoine (musée territorial) et les arts visuels (association d'enseignement de dessin, de sculpture, etc.).

\section{Les indicateurs économiques}

\section{Production}

La production est une activité exercée sous le contrôle et la responsabilité d'une unité institutionnelle qui combine des entrées - travail, capital, biens et services - pour fabriquer des biens ou fournir des services. La production est le plus souvent destinée à d'autres unités institutionnelles que l'unité productrice, mais elle peut aussi être utilisée par le producteur. On distingue trois types de production: - la production marchande, qui comprend principalement les produits vendus à des prix économiquement significatifs ;

- la production pour usage final propre, qui comprend les biens et, de façon limitative, les services produits par une unité institutionnelle pour sa propre consommation finale ou sa propre formation de capital fixe. La production de service de logement par les ménages propriétaires de leur logement appartient à cette catégorie ;

- la production non marchande, qui désigne la production destinée à d'autres unités institutionnelles, lorsqu'elle est livrée gratuitement ou à des prix sans signification économique. II s'agit ici de biens et services non vendus sur un marché car indivisibles (défense, police, éclairage public...) ou vendus à un prix très faible par volonté politique et parce qu'ils sont à l'origine d'effets externes positifs (éducation, culture). Faute de prix de marché, ces services non marchands sont évalués par la somme de leurs coûts de production: rémunération des salariés (fonctionnaires), produits utilisés comme consommation intermédiaire pour produire ces services, impôts liés à la production, consommation de capital fixe.

\section{Consommation intermédiaire}

La consommation intermédiaire est égale à la valeur des biens et des services consommés dans le processus de production. En revanche, la perte de valeur subie par les actifs fixes au cours de la production est une consommation de capital fixe. Dans certains cas, la ligne de partage entre la consommation intermédiaire et la formation de capital repose sur des conventions. C'est ainsi que font partie de la consommation intermédiaire: les travaux d'entretien courants des bâtiments des entreprises, l'achat de petit outillage, les dépenses de recherche et développement, la formation du personnel, etc.

\section{L'application des règles de la comptabilité nationale à la production des branches culturelles}

Les entreprises culturelles (maison d'édition, groupe de presse, salle de cinéma, chaîne de télévision, agence d'architecture, salle de spectacles privée, créateur artistique, etc.) produisent la très grande majorité de la production marchande (63 milliards d'euros) des branches culturelles, une partie de leur production étant par ailleurs consacrée à des biens et services non culturels (service de restauration dans une salle de spectacles par exemple). Une part très minoritaire de la production marchande culturelle (2 milliards d'euros au total) provient des entreprises dont l'activité principale n'est pas culturelle (édition de livres dans une entreprise, spectacles 
dans un restaurant) d'une part, et d'autre part, de la production marchande résiduelle de certaines administrations culturelles, vendant des produits culturels au prix du marché (vente de livres par un musée public par exemple ${ }^{1}$ ). La production marchande dans les branches culturelles est donc de 65 milliards d'euros.

L'ensemble des administrations publiques est à l'origine d'une production non marchande culturelle de 14 milliards d'euros. De leur côté, les ISBLSM, qui sont dans leur très grande majorité des associations sans but lucratif (école de musique, compagnie théâtrale, etc.) totalisent en 2011 des coûts de production de 2 milliards d'euros qui, par convention, correspondent à leur production non marchande. Les ménages bénéficient ensuite de la production non marchande culturelle des APU et des ISBLSM, en accédant à des billets d'entrée dans les musées ou les salles de spectacle ou des services d'éducation artistique (conservatoire) à des prix plus faibles. Notons que les ménages, par leurs achats de billets d'entrée ou de spectacles, contribuent en partie à faire diminuer les coûts nets de production des administrations et associations culturelles, mais par convention, ce coût n'est pas retranché dans le calcul de la production non marchande.

Enfin, les entreprises culturelles génèrent 4 milliards d'euros de production immobilisée de biens et services culturels (par exemple: programmes de télévision tournés et destinés à être diffusés ultérieurement).

Au total, la production marchande, non marchande et immobilisée des branches culturelles est donc de $65+16+4=85$ milliards d'euros.

\section{Graphique 8-La production dans les secteurs et dans les branches culturels en 2011}

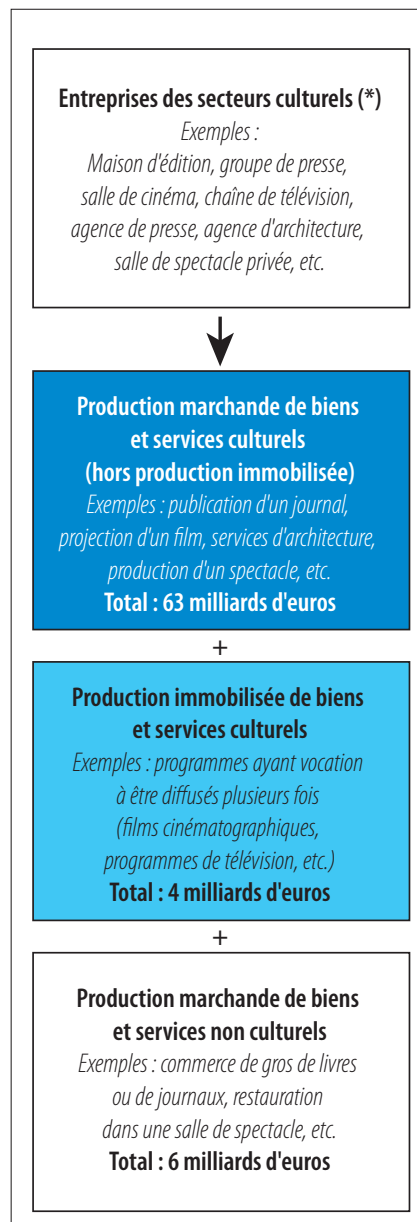

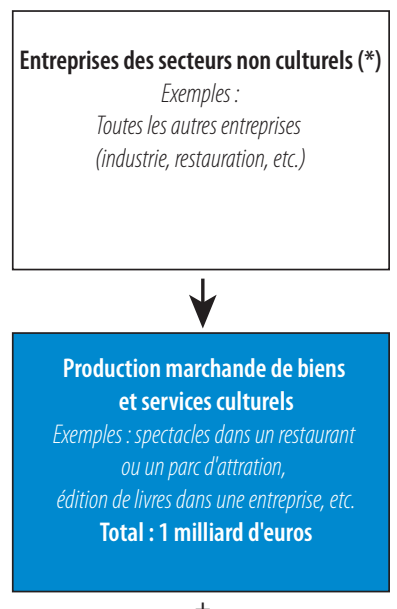

Production marchande de biens et services non culturels

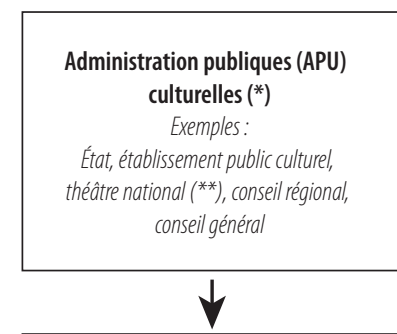

Production marchande "résiduelle" (vendue au prix du marché)

Exemples: vente de livres par un musée,

vente de lives par une administration, etc.

Total: 1 milliard d'euros

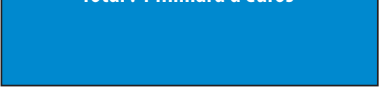

Production non marchande (vendue

à un prix inférieur à celui du marché)

évaluée au coût de production

Exemples : coût de fonctionnement d'un

établissement public, dépenses consacrées

à la culture par une collectivité territoriale, et

Total : 14 milliards d'euros

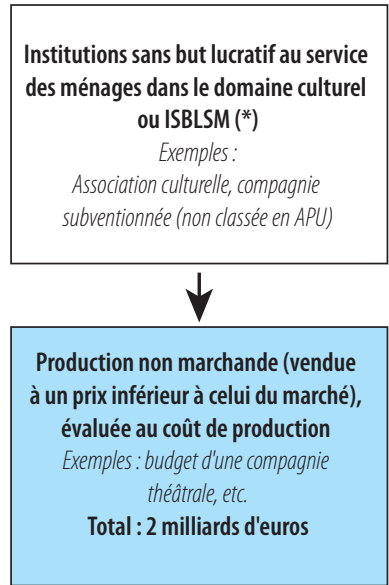

Production non marchande (vendue un prix inférieur à celui du marché),

évaluée au coût de production théâtrale, etc.

Total : 2 milliards d'euros

Note : cette décomposition est une estimation permettant de situer l'ensemble de la production culturelle. Les données sont à considérer avec précaution. $\left.{ }^{*}\right)$ Voir encadré 2

$\left.{ }^{* *}\right)$ Seuls quelques établissements subventionnés sont considérés comme des APU par la Comptabilité nationale, les autres étant des ISBLSM (voir encadré 2). 


\section{Éléments de bibliographie et données en ligne}

Champ statistique de la culture et données culturelles

DEROIN Valérie, Conceptualisation statistique du champ de la culture, Paris, Ministère de la Culture et de la Communication, DEPS, coll. «Culture méthodes », 2011-3, décembre 2011.

Cadre de l'Unesco pour les statistiques culturelles (CSC) 2009, http://unesdoc.unesco.org/images/0019/001909/190909f.pdf

Lacroix Chantal, Chiffres clés 2013. Statistiques de la culture, Paris, Ministère de la Culture et de la Communication/La Documentation française, 2013.

Pratiques et consommations culturelles

«Les services marchands en 2011. Rapport sur les comptes », Insee, document de travail, juin 2012.

Olivier Donnat, Les Pratiques culturelles des Français à l'ère numérique, enquête 2008, Paris, Ministère de la Culture et de la Communication/La Découverte, 2009.

Olivier Donnat, Les Pratiques culturelles des Français à l'ère numérique, enquête 2008, synthèse, Paris, Ministère de la Culture et de la Communication, DEPS, coll. «Culture études », 2009-6, 2009.

Chantal LACROIX, Les Dépenses de consommation des ménages en biens et services culturels et télécommunications, Paris, Ministère de la Culture et de la Communication, DEPS, coll. «Culture chiffres », 2009-2, 2009.

Bruno Maresca, Romain PICARD, Thomas PILORIn, Dépenses culture-médias des ménages au milieu des années 2000 : une transformation structurelle, Paris, Ministère de la Culture et de la Communication, DEPS, coll. « Culture études », 2011-3, 2011.

Données et documents méthodologiques sur la comptabilité nationale

Mélanie VANDERSCHELDEN, « La place du secteur associatif et de l'action sociale dans l'économie », Insee Première, juin 2011, $\mathrm{n}^{\mathrm{o}} 1356$.

Données détaillées et répartition de la valeur ajoutée par branche : http://www.insee.fr/fr/themes/theme.asp?theme=16\&sous_theme=5.2

Données détaillées sur la consommation des ménages :

http://www.insee.fr/fr/themes/theme.asp?theme=16\&sous_theme=2.3

Données détaillées sur la dépense des administrations publiques par fonction: $\mathrm{http} / / / \mathrm{www}$. insee.fr/fr/themes/theme.asp?theme=16\&sous_theme $=3.3$

Notes méthodologiques sur les concepts et les méthodes d'estimation : http://www.insee.fr/fr/themes/comptes-nationaux/default.asp?page=base_2005/methodologie/methodologie.htm

\section{ABSTRACT}

In 2011, cultural industries had a total production of 85 billion euros with an added value of 40 billion euros. Cultural production is divided and shared between a merchantable amount of 69 billion euros from the sale of companies' cultural goods and services, and a non-merchantable amount of 16 billion euros, which corresponds, by convention, to the production costs of departments, public institutions and associations in the cultural sector.

The direct economic impact of culture, measured as the relationship between the added value of cultural industries and that of all industries, was 2.2\% in 2011. It did not take into account the indirect or induced economic benefits resulting from culture (tourism, for example). Until 2003, the direct economic impact developing the value of culture has increased. However, it has been falling since 2004, which is mainly attributed to the marked decline in activity in certain cultural industries (publishing, the press, record).

In 2011, the audio-visual industry (radio, cinema, television, video, record) contributed to one quarter of added cultural value. The performing arts and heritage, predominantly non-merchantable industries, contributed to $18 \%$ and $11 \%$ of added value respectively. The progress made during the last fifteen years in the share price in terms of value of the performing arts is mainly down to the rising prices in this industry. In contrast, books and the press only contributed to $15 \%$ of added cultural value in 2011, versus $26 \%$ in 1995. Other cultural industries (advertising agencies, architecture, the visual arts, cultural education) contributed, as a whole, to one third of added cultural value.

\section{Tous les documents publiés par le DEPS sont téléchargeables sur http://www.culturecommunication.gouv.fr/Etudes-et-statistiques et sur www.cairn.info}

Pour recevoir régulièrement les publications du DEPS et pour toute demande d'information: contact.deps@culture.gouv.fr 